\title{
Los Suboficiales del Cuerpo Auxiliar de Practicantes de Farmacia Militar (1945-1989)
}

\author{
Naranjo García, JF. ${ }^{1}$
}

Sanid. mil. 2015; 71 (3): 210-213; ISSN: 1887-8571

\begin{abstract}
RESUMEN
El Ejército cuenta con dos grupos de suboficiales con el fin de auxiliar en sus labores, a los médicos y farmacéuticos. Son los Practicantes. Los de Sanidad fueron estudiados en el primer artículo. En este segundo se aborda la trayectoria de los de Farmacia. Los actuales Ayudantes de Farmacia, pertenecientes a la Escala Básica de Suboficiales, son los herederos de unos profesionales sanitarios de la milicia que, aunque tan antiguos como la propia guerra, comenzaron su andadura militar en 1945, fruto de un gran legado histórico y desarrollan hasta la actualidad, un trabajo callado dentro de la Escala de Suboficiales del Ejército. Cambiaron de nombre pero no de trabajo. Esta es su pequeña Historia.
\end{abstract}

PALABRAS CLAVE: Practicante, Farmacia Militar, Auxiliares sanitarios.

\section{Sub-officials of the Auxiliary Corps of Military Pharmacy Practitioners (1945-1989)}

SUMMARY: The army counts on two groups of NCOs to support the roles of doctors and pharmacists: the technicians. Those of the former group were studied in a previous article. This second article looks to encompass the trajectory of the latter group. The current pharmaceutical assistants, belonging to the basic NCO grade, are the descendants of militia medical professionals; although they are as old as warfare itself, their official incorporation into the military didn't come about until 1945, as the fruit of a great historical legacy and they continue into the present day, carrying out an invisible labour amongst the NCO grades. Their name has changed, but not their work. This is their little history.

KEY WORDS: Technician, Military pharmacist, Nursing auxiliary.

\section{ANTECEDENTES HISTÓRICOS}

Como en el artículo referente al Cuerpo Auxiliar de Practicantes de Sanidad Militar ${ }^{1}$, el otro Cuerpo de Sanidad profesional en el que estuvieron presentes los suboficiales fue el Cuerpo Auxiliar de Practicantes de Farmacia Militar, cuya creación y desarrollo fue parejo en el tiempo con el de ellos y siguieron trayectorias paralelas.

Su misión primordial fue la de auxiliar en sus funciones peculiares a los jefes y oficiales farmacéuticos.

Sin pretender hacer una reflexión histórica sobre los practicantes de Farmacia Militar en el Ejército, ya lo hizo de manera muy concienzuda el teniente coronel Peña Torrea en 1954, trataré de realizar un recorrido desde que fueron nombrados militares de carrera ${ }^{2}$. Los practicantes de Farmacia Militar ya aparecían en las Ordenanzas y Reglamentos de Hospitales Militares de 1739, pero nosotros arrancaremos de principio del siglo pasado cuando una real orden circular estableció las normas del reclutamiento de aquel personal, así como sus categorías: practicantes de tercera o de ingreso, de segunda y de primera, así como los mozos ${ }^{3}$. Para pertenecer a aquellas clases debían estar sirviendo en la brigada Sanitaria, podían ser elegidos los licenciados del Ejército y por último, en ausencia de

\footnotetext{
Tte. de Transmisiones del Ejército (Reserva). Doctor en Historia
}

Dirección para correspondencia: jnargar@gmail.com

Recibido: 17 de noviembre de 2014

Aceptado: 13 de febrero de 2015 los anteriores, personal civil, para lo que, además de tener la práctica necesaria para desarrollar su labor, tenían que superar unos exámenes.

Como podemos apreciar, aún no se les asignaban categorías militares, ni siquiera asimilaciones. El pase de una categoría a otra, que traía consigo un aumento de sueldo, era considerado como una gratificación por su «idoneidad y celo». Tenemos que espera a 1908 para la aparición del primer reglamento de Practicantes de Farmacia Militar, en el que, además de las categorías anteriores, aparece la de escribientes, que disfrutarían de las mismas categorías que los practicantes. Se recogía toda la legislación que sobre aquel personal existía hasta la fecha.

A partir de 1918, comienza a dársele una cierta consideración militar, aunque solo fuera a efecto de viaje, hospitalizaciones, alojamiento y raciones de campaña ${ }^{4}$. La consideración fue de sargento, pero no olvidemos que en aquella época, el sargento pertenecía a la clase de tropa, y que no fue incluido en el Cuerpo de Suboficiales creado por la República en $1931^{5}$. Fue a partir de 1934 cuando el ministro Diego Hidalgo lo integró dentro del Cuerpo de Suboficiales ${ }^{6}$.

La uniformidad de los practicantes era una blusa blanca ${ }^{7}$. En 1920 se les asignó uniforme (pantalón y chaqueta kaki) cuando estuvieran de servicio al público, y dentro del emblema las siglas P.F., relegando la blusa blanca reglamentaria al resto de las dependencias ${ }^{8}$.

Un nuevo reglamento, mucho más extenso y preciso que los anteriores, se publicó en 1929. En él se les asignó unas consideraciones militares, aunque no asimilaciones ${ }^{9}$. Así, los de tercera categoría tenían asimilaciones de suboficial -recordemos que 
suboficial era un empleo, no una categoría, que pertenecían a la segunda clase de tropa- y los de segunda y primera se les asimiló a oficiales, por lo que serían saludados por sus inferiores militares y saludarían a sus superiores.

Aquellas asimilaciones traían consigo los mismos beneficios que a los militares en lo relativo a viajes, hospitalizaciones, dietas, alojamientos etc. pero no necesitaban autorización para contraer matrimonio, como era el caso del personal militar. Los ascensos no traían consigo cambio de destino, pudiendo continuar en ellos toda su carrera si así lo deseaban. El retiro forzoso era a los 60 años de edad, pero se podía prorrogar si las necesidades así lo marcaban.

La uniformidad se estandarizó e igualó con la de los oficiales de Sanidad Militar, pero con sus símbolos correspondientes: la copa con dos cordoncillos en oro, unidos en un ángulo de $70^{\circ}$ para los de tercera categoría, cuatro cordoncillos en igual disposición, para los de segunda y seis para los de primera. En el rombo del cuello, portaban la copa de Farmacia Militar ya descrita. Estaban sujetos a las sanciones marcadas en el reglamento. $\mathrm{Y}$ se crearon nuevos escalafones atendiendo a las diferentes categorías.

Con la llegada de la Segunda República, y dentro de la profunda reorganización del Ejército llevada a cabo por Azaña, se declararon a extinguir los diversos cuerpos político-militares existentes, en uno de los cuales se encontraban precisamente los Practicantes de Farmacia Militar. Se creó un nuevo Cuerpo que los aglutinó, denominado Cuerpo Auxiliar de Subalternos del Ejército (CASE) ${ }^{10}$.

De las cinco Secciones independientes con las que se creó el nuevo Cuerpo, los Practicantes de Farmacia Militar se integraron en la Segunda, denominada Subalternos Periciales, al igual que los Practicantes de Sanidad Militar. El ingreso en el nuevo Cuerpo era voluntario, quedando el resto a extinguir. Se les computó, a efectos de sueldo, el tiempo de servicio.

En estos momentos, tampoco poseían asimilación militar, aunque si consideración de oficial o suboficial, dependiendo del sueldo. Tenían un uniforme común las tres primeras Secciones, pero sin las insignias análogas o similares que los oficiales, llevando las del $\mathrm{CASE}^{11}$.

Durante la Guerra Civil, dada la escasez de este personal, fue necesario incorporarlos en ambos bandos, aunque fue el Nacional el que lo legisló de manera más precisa ${ }^{12}$.

Una vez finalizada la Guerra Civil, se comenzó el desmantelamiento progresivo del CASE, y se crearon una serie de Cuerpos de Suboficiales que lo sustituyeron en sus diferentes especialidades. Fue así como se creó en 1945, el Cuerpo Auxiliar de Practicantes de Farmacia Militar ${ }^{13}$.

\section{Constitución y Pruebas de Acceso.}

El nuevo Cuerpo se constituyó con los Practicantes de Farmacia Militar pertenecientes al CASE que así lo solicitaron y con los componentes del extinto Cuerpo político-militar de Practicantes de Farmacia Militar, que en el momento de su desaparición no quisieron integrarse en aquél cuando se creó en 1932. A partir de entonces sería por acceso directo con oposición, pero dando preferencia a los sargentos y tropa que demostraran más de un año de prácticas en Farmacias Militares. El personal CASE, que en el momento de la integración tuviera la consideración de oficial, la conservaría aunque no le correspondiera en el nuevo escalafón, hasta alcanzarla con el tiempo.

La primera oposición se convocó el año 1946 con las siguientes condiciones ${ }^{14}$ :

- Militares, no tener cumplidos los cuarenta años de edad.

- Civiles, treinta y un años de edad, además de certificar las prácticas en farmacias o aprobados tres años de bachillerato.

- Las condiciones físicas adecuadas para el desarrollo de su función.

Los exámenes consistían en cinco pruebas:

- Reconocimiento médico: era calificado con "apto" o "no apto".

- Reconocimiento de medicamentos, material y productos químicos-farmacológicos, elaboración de fórmulas magistrales etc.

- Desarrollo oral de uno de los temas de física, química, historia natural y farmacia práctica de los que figuraban en el programa.

- Resolución de dos temas de aritmética y dos de geometría.

- Desarrollo por escrito de un tema de geografía y otro de historia, para acreditar suficiencia en la redacción.

Menos la primera, las pruebas debían ser aprobadas al menos con un cinco y eran eliminatorias estableciéndose la ponderación correspondiente. Una vez finalizadas las pruebas se publicaban las actas y se escalafonaban de acuerdo con la puntuación obtenida.

Nuevas instrucciones para las oposiciones se publicaron diez años después ${ }^{15}$. En realidad, se trataba de las primeras ya que las anteriores se basaban en las publicadas en cada convocatoria. En ellas se establecieron las siguientes condiciones para el ingreso:

- Civiles y militares con edades no superiores a los 39 años de edad (30 para los primeros).

- Poseer aptitud psicofísica.

- Llevar un año de Servicio Militar, y para los civiles tenerlo cumplido.

- Para los civiles, haber realizado al menos un año de prácticas en farmacia, o tener tres años de bachillerato.

- Derecho de examen (40 pesetas) de la que estarían exentos los militares.

El resto de las condiciones y pruebas de acceso variaron poco.

\section{PERIODO DE FORMACIÓN}

Los que superaban la oposición, ingresaban en la Academia de Farmacia Militar para realizar un curso de cuatro meses, en régimen de internado, con las siguientes materias:

- Organización militar y especialmente de Farmacia Militar 
- Ordenanzas, régimen interior y justicia militar

- Táctica, servicios farmacéuticos militares, deontología, contabilidad y documentación farmacéutica, nomenclátor y petitorios de Farmacia Militar.

- Prácticas bajo la inspección del profesorado en los laboratorios y en el Parque Central de Farmacia, en Farmacias Militares de plaza y hospitales de Madrid.

\section{MODELO DE CARRERA}

Los que superaban el curso eran promovidos a practicantes de tercera, asimilados a sargento, y se escalafonaban atendiendo a la puntuación obtenida. Se establecieron los empleos y plantillas iniciales siguientes, teniendo en cuenta que los empleos del Cuerpo de Suboficiales en aquella época eran las de sargento y brigada:

- Practicantes de primera, asimilados a teniente: 24.

- Practicantes de segunda, asimilados a brigada: 47.

- Practicantes de tercera, asimilados a sargento: 45.

Los ascensos a los empleos superiores se establecieron por riguroso orden de antigüedad en el empleo, sin defecto, con ocasión de vacante y la declaración de aptitud, al cumplir los tres años en sus empleos. Los que ingresaron del CASE, y que conservaron la consideración de oficial, al llevar más de tres años, se pensó que tenían ya consolidada la efectividad para el ascenso superior, en las mismas condiciones expresadas ${ }^{16}$.

En 1965 se aplicó al Cuerpo lo establecido en la ley de 1960 por la que se creaban dos nuevos empleos en el Cuerpo de Suboficiales del Ejército: sargento primero y subteniente por lo que las asimilaciones a los empleos quedaron ${ }^{17}$ :

- Practicantes de segunda: brigada y subteniente.

- Practicantes de tercera: sargento y sargento primero.

Los ascensos a sargento primero se alcanzarían al cumplir 10 años de servicio en el empleo de sargento y para ascender a subteniente, los mismos en el empleo de brigada. Para el ascenso a brigada y teniente no se estableció tiempo de mínima permanencia. Se fijó la edad de retiro a los sesenta años.

\section{EVOLUCIÓN DEL CUERPO AUXILIAR DE PRACTICANTES DE FARMACIA MILITAR}

En 1974 se creó la Escala Básica de Suboficiales (EBS) y la Especial de Jefes y Oficiales (EE) del Ejército ${ }^{18}$. En ella se declaró a extinguir el Cuerpo Auxiliar de Practicantes de Farmacia Militar, aunque se les dio la opción de ingresar en la nueva Escala creada o continuar en la suya. Del mismo modo, podían optar por ingresar en la Escala Especial de Jefes y Oficiales con el título de bachiller elemental, si bien, los así ingresados no podrían ascender a capitán hasta no haber obtenido el de bachiller superior o BUP.

En la nueva EBS se creó, dentro de los Especialistas, la Rama de Farmacia, en donde volvió a aparecer este grupo de profesionales militares como herederos de los Practicantes de Farmacia $^{19}$. Éstos, además del emblema de todos los Especialistas de la
EBS en los rombos del cuello de la guerrera ${ }^{20}$, llevaban el de su propia especialidad en el brazo derecho de la guerrera: La copa con la serpiente enrollada ${ }^{21}$. Desarrollaron su formación en el Instituto Politécnico del Ejercito (IPE) n ${ }^{\circ} 2$, en Calatayud (Zaragoza) y eran denominados genéricamente FAF.

No hay que confundir la Rama de Farmacia, pertenecientes a los Especialistas de la EBS, con los miembros del Cuerpo de Farmacia, de los Cuerpos de la EBS. Éstos últimos tenían como misión el mando de tropas de unidades de Farmacia Militar. Llevaban las insignias propias de la Farmacia: el cáliz con la áspid y las ramas de olivo.

Como consecuencia de la promulgación de la ley de reorganización de las EBS y EE ${ }^{22}$, se estableció que los oficiales especialistas de la EE tendrían un único escalafón, por lo que se publicaron las especialidades correspondientes, en la que estaba representado los Ayudantes de Farmacia ${ }^{23}$.

Con la entrada en vigor de la primera ley del personal militar de los tres Ejércitos y Cuerpos Comunes, desapareció la especialidad de Auxiliar de Farmacia Militar, integrándose en la Escala Básica del Cuerpo de Especialistas del Ejército, tanto los pertenecientes al Cuerpo de Suboficiales de Farmacia como a la Escala Básica de Suboficiales de Farmacia. Se estableció que no se producirían más ascensos en las escalas declaradas a extinguir a no ser que lo solicitaran con el pase a la situación de reserva ${ }^{24}$.

Para finalizar, en 1999 se promulgó una nueva ley del Régimen del Personal Militar en la cual se estipuló que los subtenientes y brigadas del Cuerpo Auxiliar de Practicantes de Farmacia, que aún no hubieran pasado a la situación de reserva o reserva transitoria y que reunieran las condiciones para ello, podrían solicitar su integración en las Escalas Auxiliares con el empleo de teniente, con ocasión de vacante ${ }^{25}$.

Como consecuencia de la incorporación de la mujer a las Fuerzas Armadas, a partir de 1989, los Auxiliarles de Farmacia Militar, tuvieron representación femenina, tal y como indicaba el real decreto ley ${ }^{26}$. La última promoción de la EB en las que estuvieron presentes este colectivo militar profesional fue en la XVIII, que se convocó en el año 1991 y en la que también hubo representación femenina ${ }^{27}$.

Estas líneas son un homenaje a ese personal militar que siempre ha realizado una labor callada y ejemplar dentro del Ejército.

\section{BIBLIOGRAFÍA}

1. Naranjo García J.F. Revista de Sanidad Militar 2014; 70(3):215-217.

2. Peña Torrea, F. El servicio de Farmacia Militar en pie de paz, Diario Oficial del Ejército 1954:61-71.

3. Real orden circular por la que se establecen las categorías y reclutamiento de los Practicantes de Farmacia Militar, de 12 de septiembre de 1905: DOE. n. ${ }^{\circ} 202$. Se aclara por real orden circular de 5 de diciembre del mismo año: DOE. 272.

4. Real orden circular por la que se les asigna la consideración de sargento para pasaje, hospitalizaciones, alojamientos etc., de 29 de diciembre de 1918: DOE. $n .^{\circ} 1$.

5. Ley por la que se crea el Cuerpo de Suboficiales del Ejército, de 4 de diciembre de 1931: GM. n. ${ }^{\circ} 339$.

6. La incorporación del sargento en el Cuerpo de Suboficiales fue como consecuencia de la ley de reorganización del Cuerpo de Suboficiales, de 5 de julio de 1934: GM. n. ${ }^{\circ} 193$.

7. Artículo 26 de la real orden circular de 6 de octubre de 1920, reglamento de régimen interno de las farmacias de hospitales militares y servicio especial relativo a la venta de medicamentos: CLE. n. ${ }^{\circ} 247$. 


\section{Los Suboficiales del Cuerpo Auxiliar de Practicantes de Farmacia Militar (1945-1989)}

8. Real orden circular por la que se establece la uniformidad de los Practicantes de Farmacia Militar cara al público, de 25 de marzo de 1926. Colección legislativa del Ejército (CLE) n. ${ }^{\circ} 121$. El emblema de Farmacia Militar se estableció por real orden circular de 14 de junio de 1919: CLE. n. ${ }^{\circ} 114$.

9. Real orden circular por la que se aprueba el reglamento de los Practicantes Militares de Farmacia, de 31 de julio de 1929: DOE. 169.

10. Ley de 13 de mayo de 1932, por la que se crea el Cuerpo Auxiliar Subalternos del Ejército: GM. n. ${ }^{\circ} 136$.

11. Orden por la que se dispone la uniformidad del personal de las tres primeras Secciones del CASE, de 24 de junio de 1933: DOE. 146.

12. Orden por la que se autoriza la incorporación como Practicantes de Farmacia, al personal que hubiera ejercido como tal en la vida civil o cursado al menos dos años de la carrera de Farmacia, de 12 de marzo de 1937: BOE. n. ${ }^{\circ} 145$.

13. Ley por la que se crea el Cuerpo Auxiliar de Practicantes de Farmacia Militar, de 17 de julio de 1945: BOE. n. ${ }^{\circ} 202$. Se desarrolló por orden de 30 de agosto de 1945: DOE. n. ${ }^{\circ} 196$.

14. Orden por la que se convoca oposición para el ingreso en el Cuerpo de Practicantes de Farmacia Militar, 10 de abril de 1946: DOE n. ${ }^{\circ} 89$.

15. Orden por la que se dan instrucciones para las convocatorias de ingreso en el Cuerpo Auxiliar de Practicantes de Farmacia Militar, de 6 de junio de 1956: BOE. $n .^{\circ} 174$

16. Orden de 14 de junio de 1946: CLE. n. ${ }^{\circ} .107$.

17. Orden por la que se establecen los nuevos empleos de la ley 21 de julio de 1960 al Cuerpo Auxiliar de Practicantes de Farmacia, 19 de julio de 1965: DOE n. ${ }^{\circ} 112$
18. Ley 13/1974, de 30 de marzo, de organización de las Escalas Básicas de Suboficiales y Especial de Jefe y Oficiales del Ejército: BOE. n. ${ }^{\circ} 78$. Se desarrolló por decreto 2956/1974, de 27 de septiembre: BOE 258.

19. Orden por que se disponen las ramas de la Escala Básica de Suboficiales Especialistas y sus especialidades, de 26 de junio de 1975 : DOE. n. ${ }^{\circ} 152$.

20. Orden por la que se crea el emblema y rombo portaemblemas para las Escalas Especial de Jefes y Oficiales Especialistas y Básica de Suboficiales Especialistas, de 11 de septiembre de 1975: DOE. n. ${ }^{\circ} 214$.

21. Punto 2.2.9. de la orden por la que se crean los distintivos de especialidad de la Escala Básica de Suboficiales Especialistas y Especial de Jefes y Oficiales, de 28 de junio de 1977: DOE. n. ${ }^{\circ}$ 154. Fue modificado por orden de 13 de junio de 1979: DOE. n. ${ }^{\circ} 133$.

22. Ley $14 / 1982$, de 5 de mayo, por la que se reorganizan las Escalas Especial y Básica del Ejército de Tierra: BOE. n. ${ }^{\circ} 115$.

23. Ley $11 / 1984$, de 12 de abril, por la que se determinan las especialidades de la Escala de Jefes y Oficiales Especialistas del Ejército de Tierra: BOE. n. ${ }^{\circ}$ 92. Era la especialidad en la que se integrarían los suboficiales Ayudantes de Farmacia que promocionaran.

24. Ley 17/1989, de 19 de julio, reguladora del Régimen del Personal Militar Profesional: BOE. n. ${ }^{\circ} 172$.

25. Ley $17 / 1999$, de 18 de mayo, de Régimen de Personal de las Fuerzas Armadas: BOE. n. ${ }^{\circ} 119$.

26. Real decreto ley 1 , de 22 de febrero, por el que se regula la incorporación de la mujer a las Fuerzas Armadas: BOE. n. ${ }^{\circ} 46$.

27. Resolución 442/38702/1991. De 26 de abril: BOD. n. ${ }^{\circ} 88$. 